\title{
The role of laparoscopy in the surgical treatment of endometrial cancer
}

\author{
Paweł S. Pawłowicz ${ }^{1}$, Urszula Ajdacka² \\ ${ }^{1}$ Department of Obstetrics and Gynecology, Rural Hospital, Garwolin, Poland \\ ${ }^{2}$ Clinic of Obstetrics and Gynecology, Mother and Child Institute, Warsaw, Poland
}

Videosurgery Miniinv 2015; 10 (1): 44-48

DOI: $10.5114 /$ wiitm.2015.49249

\begin{abstract}
Introduction: Endometrial carcinoma is one of the most common neoplasms in gynecological oncology and the most common genital cancer in women in developed countries. The 5-year overall survival rate depends on the FIGO stage. For patients with stage I endometrial cancer it is estimated as $80 \%$. Traditionally, the main treatment of endometrial cancer consists of total abdominal hysterectomy with bilateral salpingo-oophorectomy and, in some histological or clinical stages, with additional pelvic lymphadenectomy. The main surgical approach so far for women with endometrial cancer has been laparotomy. However, in the last decades there have been many reports stating that the survival rate in such cases is similar after laparoscopy and laparotomy. Some researchers claim that laparoscopy is as effective as laparotomy, and it might be much more precise than laparotomy thanks to its special optic system.

Aim: To establish the method of choice for treatment of stage I endometrial cancer.

Material and methods: Comparing the preliminary results from data collected during surgical procedures performed at the First Department of Obstetrics and Gynecology of the Medical Centre of Postgraduate Education in Warsaw and the Department of Obstetrics and Gynecology of the Rural Hospital in Garwolin.

Results: Laparoscopy is as effective as laparotomy, and it might be much more precise than laparotomy.

Conclusions: Taking into consideration all the above arguments, if an experienced endoscopic surgeon and proper laparoscopic equipment are available, laparoscopy might become the method of choice for treatment of stage I endometrial cancer.
\end{abstract}

Key words: laparoscopy, endometrial cancer, lymphadenectomy.

\section{Introduction}

Endometrial cancer is the most common genital cancer in women in developed countries. The mean age of diagnosis of endometrial cancer is 60 years. A distinction is made between two types of endometrial cancer. Type I (endometrioid, 80-90\%) represents estrogen-dependent tumors responding to treatment with progestogens. It is associated with good prognosis (the total 5 -year survival rate is 75-85\%). Type II (non-endometrioid, 10-20\%) may be independent from unrestrained estrogen stimulation. Tumors of type II are poorly differentiated and aggressive histologically (carcinoma, papillary serous) and are associated with a lower total 5-year survival rate (35\%) [1].

Most patients can be diagnosed at an early stage of the disease. Often, the first manifestation is abnormal vaginal bleeding, especially in postmenopausal women. Bleeding is diagnosed with fractional curettage or aspiration biopsy [2]. There are four histological types of endometrial hyperplasia: simple hyperplasia, com-

\section{Address for correspondence}

Urszula Ajdacka MD, Clinic of Obstetrics and Gynecology, Mother and Child Institute, 17a Kasprzaka St, 01-211 Warsaw, Poland,

phone: +48 502215 096, e-mail: urszula.ajdacka@gmail.com 
plex hyperplasia, simple hyperplasia with atypia and complex hyperplasia with atypia. In diagnosis of complex hyperplasia with atypia there is a possibility of progression to endometrial carcinoma or coexistence of endometrial cancer. Numerous studies have shown that the risk of coexistence of complex endometrial hyperplasia and endometrial cancer is high and may reach even $20-50 \%$ of cases $[2,3]$. This risk must be taken into account when considering therapeutic options. Complex hyperplasia with atypia, confirmed in the final pathological examination after hysterectomy, requires no further treatment [4].

The prognosis for patients depends on the stage of cancer according to the FIGO staging system. There is a good prognosis in FIGO stage I, when the tumor is limited to the uterus. The overall 5-year survival rate for these patients is estimated to be about $80 \%$ [5-7]. The main surgical approach to the treatment of endometrial cancer in FIGO stage I is a hysterectomy with bilateral salpingo-oophorectomy, which is traditionally performed by laparotomy. This procedure should be extended to additional pelvic lymphadenectomy in some specific cases. They are as follows: moderately or poorly differentiated (grade G2 or G3 cancer), clear cell or serous cancer, and when the infiltration through the myometrium is over $50 \%$, although accurate indications for these procedures are not clear and are still controversial $[5,8,9]$. The latest studies have revealed that this procedure might be performed by laparoscopy with similar efficacy to laparotomy. Furthermore, many researchers have noted some advantages of laparoscopic management over traditional laparotomy, especially for old and obese women [10-12]. Laparoscopic surgery for endometrial cancer was first reported in 1992 by Childers and Surwit [13]. According to some prospective studies the recurrence and survival rates among patients who underwent laparoscopic treatment of endometrial cancer seems to be similar to those observed among patients after laparotomy [14-16]. Many authors have reported that dissection of lymph nodes (LN) by a less invasive method, i.e. laparoscopy, is connected with a better postoperative course. Furthermore, higher precision during laparoscopy is observed as a result of a special optic system which gives a surgeon an enlarged view $[17,18]$. Additionally, short duration of hospitalization, less blood loss and fast convalescence after laparoscopic surgery allow patients to begin adjuvant therapy more quickly [19].

\section{Aim}

The aim of the study was to establish the method of choice for treatment of stage I endometrial cancer.

\section{Material and methods}

A retrospective review of medical records of 56 patients who underwent surgical treatment of stage I endometrial cancer at the First Department of Obstetrics and Gynecology of the Medical Centre of Postgraduate Education in Warsaw and the Department of Obstetrics and Gynecology of the Rural Hospital in Garwolin from 30 ${ }^{\text {th }}$ September 2010 to $28^{\text {th }}$ October 2014 was conducted. The first group comprised 30 patients who underwent pelvic laparoscopic lymphadenectomy, and the second group comprised 26 patients who were treated traditionally by laparotomy. The mean age of patients from the first group was $67 \pm 3$ years, and the mean age of patients from the second group was $66 \pm 9$ years. In laparoscopy the access to the retroperitoneal space and to the obturator space was achieved by identifying the triangle between the infundibulopelvic ligament, the round ligament and the external iliac artery. Afterwards, the peritoneum overlaying the common iliac arteries was opened. The incision was extended to the bifurcation of the common iliac artery into the internal and external iliac artery towards the prevesical space. The round ligament was cut and the following lymph nodes were removed en bloc: common iliac LNs, external iliac LNs, internal iliac LNs and obturator LNs. The removal of lymph nodes was conducted with graspers, bipolar scissors or a harmonic knife. All the LNs were placed in Endobags and were removed. All the operations were performed by Paweł S. Pawłowicz MD.

\section{Results}

In the first group, 6 women were diagnosed with clear cell carcinoma, in 10 cases the diagnosis was cancer of endometrioid type grade 2 , in 7 cases cancer of endometrioid type grade 3 , and in 7 cases serous carcinoma was diagnosed. Among the second group there were 6 cases of clear cell carcinoma, 5 cases of serous carcinoma, 9 cases of endometrioid cancer grade 2 and 6 cases of endometrioid cancer grade 3 . The number of lymph nodes obtained in the first group was $26 \pm 3$ and in the second group 16 \pm 4 . The average blood loss during laparoscopy was 
$438 \mathrm{ml}$ and after laparotomy it was estimated to be about $601 \mathrm{ml}$. In the first group there were 3 cases requiring antibiotic treatment in the postoperative period due to fever that was probably a result of intraperitoneal small hematomas (less than $5 \mathrm{~cm}$ diameter in sonic examination). There were also $2 \mathrm{ep}$ isodes of fever up to $38^{\circ} \mathrm{C}$ on the first day after the surgery; the fever disappeared spontaneously. After laparotomy, there were 13 cases of antibiotic treatment as a result of wound dehiscence in 6 cases, and in 7 cases there was fever up to $37.8^{\circ} \mathrm{C}$ which did not disappear spontaneously. The average length of hospitalization also differed widely between these two groups. A short hospital stay (3.3 days) was achieved for patients treated by laparoscopy, while patients after laparotomy spent about 9.6 days in the hospital.

Histopathological examination of collected materials - iliac and obturator LNs revealed in the first group: 11 cases of inflammatory changes and 9 cases of subcapsular metastasis in 13 obturator lymph nodes. Among patients who underwent laparotomy there were 11 cases of inflammatory changes and 2 cases of subcapsular metastasis in the 2 obturator lymph nodes. There were no pathological changes in the rest of the LNs. All patients after histopathological diagnosis were finally referred to the Cancer Centre and Institute of Oncology for consultation or further treatment [20].

\section{Discussion}

This comparison of pelvic lymphadenectomy performed by laparoscopy and laparotomy in stage I endometrial cancer shows that laparoscopy seems to be a more precise method than laparotomy [10, 14]. On average there are seven more LNs collected after laparotomy in comparison to LNs collected during laparoscopic lymphadenectomy. There was a better postoperative course after laparoscopy than laparotomy. Probably, it was a result of less estimated blood loss, less postoperative pain and rare antibiotic treatment. The great advantage of the laparoscopic approach is also a shorter time of hospitalization, which was about 3.3 days in comparison with 9.6 days which patients spent in the hospital after laparotomy. These results are comparable to the studies underlining the better outcomes of laparoscopic management of endometrial cancer which have been published recently $[11,12,15]$.
In July 2010 a randomized trial about the safety of laparoscopy versus laparotomy in early-stage endometrial cancer was published by Mourits. This randomized trial was done in 21 hospitals in the Netherlands, and 26 gynecologists with proven sufficient skills in total laparoscopic hysterectomy (TLH) participated. Two hundred and eighty-three patients with stage I endometrioid adenocarcinoma or complex atypical hyperplasia were randomly allocated $(2: 1)$ to the intervention group (TLH, $n=187$ ) or control group (TAH, $n=96)$. The proportion of major complications was $14.6 \%$ (27 of 185) in the TLH group vs. $14.9 \%$ (14 of 94 ) in the total abdominal hysterectomy (TAH) group, with a difference of $-0.3 \%$. The proportion of patients with an intraoperative major complication (9 of 279 (3.2\%)) was lower than the proportion with a postoperative major complication (32 of $279(11.5 \%)$ ) and did not differ between TLH and TAH. The proportion of patients with a minor complication was $13.0 \%$ (24 of 185) in the TLH group and $11.7 \%$ (11 of 94 ) in the TAH group. The result was that TLH (done by skilled surgeons) was beneficial in terms of a shorter hospital stay, less pain, and quicker resumption of daily activities [21].

In 2009 Zullo et al. published "Laparoscopic surgery versus laparotomy for early stage endometrial cancer: long-term data of a randomized controlled trial". The purpose of the study was to compare the long-term safety and efficacy of laparoscopic surgery and laparotomy approaches to early stage endometrial cancer. This was a prospective longterm extension study of a randomized controlled study that included 84 patients with clinical stage I endometrial cancer (laparoscopic surgery group, 40 women; laparotomy group, 38 women). Safety and efficacy data were evaluated and analyzed by the intention-to-treat principle. After a follow-up period of 78 months for laparoscopic surgery and laparotomy groups, respectively, no difference in the cumulative recurrence rates $(8 / 40$ (20.0\%) and $7 / 38$ (18.4\%); $p=0.860)$ and deaths (7/40 (17.5\%) and 6/38 (15.8\%) patients; $p=0.839)$ was detected between groups. No significant differences in overall $(p=0.535)$ and disease-free ( $p=0.512$ ) survival were observed. The laparoscopic surgery approach to early stage endometrial cancer is as safe and effective a procedure as the laparotomy approach [22].

In 2013 Terai et al. published a report to determine the feasibility and safety of total laparoscopic modified radical hysterectomy (TLMRH) in the treat- 
ment of presumed stage I endometrial cancer. The patients in the TLMRH group had less blood loss, a similar number of lymph nodes removed, less need for analgesia and a shorter hospital stay, but longer operations than those treated by laparotomy [23].

Lu et al. compared the laparoscopic approach with the conventional laparotomy approach for the treatment of patients with endometrial carcinoma in a developing country. They also came to the conclusion that laparoscopic surgery is a safe and reliable alternative to laparotomy in the management of endometrial carcinoma patients, with significantly reduced hospital stay and postoperative complications [24].

Bogani et al. studied laparoscopic staging in women older than 75 years with early-stage endometrial cancer in comparison with an open surgical operation. Their findings suggest that patients with endometrial cancer older than 75 years may benefit from minimally invasive surgery and should not be denied laparoscopy based on mere chronological age [25].

Yavuzcan et al. suggested that comprehensive research on a larger scale is needed in order to investigate safer and easy-to-perform methods in laparoscopic hysterectomy that would reduce urinary tract complications and all-cause complication rates [26].

The lower post-operative complication rates compared to the classical laparotomic approach and the possibility of laparoscopic treatment whenever the conditional requirements (surgeon and instruments) are met, were noticed by Ceccaroni et al. [27].

\section{Conclusions}

Evaluation of lymph nodes is an important prognostic factor in cancer of the uterus. Therefore, the removal of nodes is a necessary part of the staging of the disease and sometimes may have therapeutic relevance and improve the prognosis after surgery. Currently it is believed that laparoscopic lymphadenectomy is a method of choice, allowing for better visualization, reduced complications and lower invasiveness compared to laparotomy [28-30]. According to publications on coexistent endometrial cancer in patients with atypical endometrial hyperplasia, the majority of cancers are well-differentiated, early-staged malignancies and are most likely considered to be at low risk for lymph node metastasis [31-33]. However, not all patients have low-risk disease. Studies confirm the presence of both high- and low-risk cancers in patients with atypical endometrial hyperplasia [2, 20, 30, 33, 34].

Because of the coexistence of high-risk endometrial cancer in women with a typical endometrial hyperplasia complex and low usefulness of intra-operative tests in detecting tumor invasion, Whyte et al. suggested the usefulness of routine ilio-obturator lymphadenectomy in patients with a preoperative diagnosis of complex hyperplasia with atypia. They found that the information obtained by lymphadenectomy carried out simultaneously with hysterectomy had an impact on the choice of treatment approaches in 7 of 25 (28\%) patients with cancer. The duration of the operation was not significantly prolonged (16 $\mathrm{min})$, the blood loss was not increased and the time of hospitalization was not prolonged [35].

Taking into consideration all above arguments, if an experienced endoscopic surgeon and proper laparoscopic equipment are available, laparoscopy might become the method of choice for treatment of stage I endometrial cancer $[9,12,16]$. The results of our own studies also confirm this treatment strategy.

\section{Conflict of interest}

The authors declare no conflict of interest.

\section{References}

1. Sonoda Y, Barakat RR. Screening and prevention of gynecologic cancer: endometrial cancer. Res Clin Obstet Gyn 2006; 20 : 363-77

2. Trimble CL, Kauderer J, Zaino R, et al. Concurrent endometrial carcinoma in women with a biopsy diagnosis of atypical endometrial hyperplasia: a Gynecologic Oncology Group study. Cancer 2006; 106: 812-9.

3. Bilgin T, Ozuysal S, Ozan H, Atakan T. Coexisting endometrial cancer in patients with a preoperative diagnosis of atypical endometrial hyperplasia. J Obstet Gynaecol Res 2004; 30: 205-9.

4. Leitao MM Jr, Han G, Lee LX, et al. Complex atypical hyperplasia of the uterus: characteristics and prediction of underlying carcinoma risk. Am J Obstet Gynecol 2010; 203: 349.e1-6.

5. Kitchener HC, Trimble EL; the Endometrial Cancer Working Group of the Gynecologic Cancer Intergroup. Endometrial Cancer State of the Science Meeting. Int I Gynecol Cancer 2009; 19: 134-40.

6. Rodriguez M. Endometrial cancer: Part 1 - Epidemiology, diagnosis and work-up. Menopause Management 2001; 10: 19-21.

7. Purdie DM, Green AC. Epidemiology of endometrial cancer. Best Pract Res Clin Obstet Gynaecol 2001; 15: 341-5.

8. German Working Group on Gynaecologic Oncology. Recommendations for diagnosis and treatment in patients with endometrial carcinoma. Zentralb Gynakol 2002; 1: 58-62. 
9. ASTEC study group, Kitchener H, Swart AM, Qian Q, et al. Efficacy of systematic pelvic lymphadenctomy in endometrial cancer (MRC ASTEC trial): a randomised study. Lancet 2009; 373: 125-36.

10. Liauw L, Chung YN, Tsoi CW, et al. Laparoscopy for the treatment of women with endometrial cancer. Hong Kong Med I 2003; 9: 108-12.

11. Litta P, Fracas M, Pozzan C, et al. Laparoscopic management of early stage endometrial cancer. Eur J Gynaecol Oncol 2003; 24: 41-4.

12. Holub Z, Bartos P, Dorr A, et al. The role of laparoscopic hysterectomy and lymph node dissection in treatment of endometrial cancer. Eur J Gynaecol Oncol 1999; 20: 268-71.

13. Childers JM, Surwit EA. Combined laparoscopic and vagina surgery for the management of two cases of stage I endometrial cancer. Gynecol Oncol 1993; 51: 33-8.

14. Holub Z, Jabor A, Bartos P, et al. Laparoscopic surgery for endometrial cancer: long-term results of multicentric study. Eur J Gynaecol Oncol 2002; 23: 305-10.

15. Yavuzcan A, Yıldız G, Çağlar M, et al. Which one is safer - performing a laparoscopic hysterectomy with a tissue fusion device involving diagnostic cystoscopy or traditional abdominal hysterectomy with ureteral dissection? Videosurgery Miniinv 2013; 8: 280-8.

16. Tozzi R, Malur S, Koehler C, Schneider A. Laparoscopy versus laparotomy in endometrial cancer: first analysis of survival of a randomized prospective study. I Minim Invasive Gynecol 2005; 12: 130-6.

17. Kohler C, Klemm P, Shcau A, et al. Introduction of transperitoneal lymphadenectomy in a gynecologic oncology center: analysis of 650 laparoscopic pelvic and/or paraaortic transperitoneal lymphadenectomies. Gynecol Oncol 2004; 95: 52-61.

18. Sobiczewski P, Bidziński M, Derlatka P, et al. Comparison of the results of surgical treatment in patients with endometrial cancer. Int J Gynecol Cancer 2005; 15: 946-51.

19. Kehoe S, Abu-Rustum N. Transperitoneal laparoscopic pelvic and paraaortic lymphadenectomy in gynecologic cancers. Curr Treat Oncol 2006; 7: 93-101.

20. Pawłowicz P, Grzebyk K, Stetkiewicz T, et al. A comparison of laparoscopy and laparotomy for the pelvic lymphadenectomy in endometrial cancer at the I Department of Obstetrics and Gynaecology of Medical Centre of Postgraduate Education in Warsaw - own preliminary experience. Prz Menopauz 2011; 15: 114-5.

21. Mourits M, Bijen C, Arts H, et al. Safety of laparoscopy versus laparotomy in early-stage endometrial cancer: a randomised trial. Lancet Oncol 2010; 11: 763-71.

22. Zullo F, Palomba S, Falbo A, et al. Laparoscopic surgery vs. laparotomy for early stage endometrial cancer: long-term data of a randomized controlled trial. Am J Obstet Gynecol 2009; 200: 296.e1-9.

23. Terai Y, Tanaka T, Sasaki H et al. Total laparoscopic modified radical hysterectomy with lymphadenectomy for endometrial cancer compared with laparotomy. J Obstet Gynaecol Res 2014; 40: 570-5.

24. Lu Q, Liu H, Liu C, et al. Comparison of laparoscopy and laparotomy for management of endometrial carcinoma: a prospective randomized study with 11-year experience. I Cancer Res Clin Oncol 2013; 139: 1853-9.
25. Bogani G, Cromi A, Uccella S, et al. Laparoscopic staging in women older than 75 years with early-stage endometrial cancer: comparison with open surgical operation. Menopause 2014; 21: 945.

26. Yavuzcan A, Yıldız G, Çağlar M, et al. Which one is safer - performing a laparoscopic hysterectomy with a tissue fusion device involving diagnostic cystoscopy or traditional abdominal hysterectomy with ureteral dissection? Videosurgery Miniinv 2013; 8: 280-8.

27. Ceccaroni M, Roviglione G, Pesci A, et al. Total laparoscopic hysterectomy of very enlarged uterus (3030 g): case report and review of the literature. Videosurgery Miniinv 2014; 9: 302-7.

28. Gil-Moreno A, Díaz-Feijoo B, Morchón S, et al. Analysis of survival after laparoscopic-assisted vaginal hysterectomy compared with the conventional abdominal approach for early-stage endometrial carcinoma: a review of the literature. J Minim Invasive Gynecol 2006; 13: 26-35.

29. Martinek I, Haldar K, Tozzi R. Laparoscopic surgery for gynaecological cancers in obese women. Maturitas 2010; 65: 320-4.

30. Pawłowicz P, Czekańska M, Rawski W, et al. Laparoscopic excision of pelvic lymphs nodes after vaginal hysterectomy due to histological blunder. Case report of two patients. Prz Menopauz 2011; 5: 383-5.

31. Hunter JE, Tritz DE, Howell MG, et al. The prognostic and therapeutic implications of cytologic atypia in patients with endometrial hyperplasia. Gynecol Oncol 1994; 55: 66-71.

32. Widra EA, Dunton CJ, McHugh M, et al. Endometrial hyperplasia and the risk of carcinoma. Int J Gynecol Cancer 1995; 5: 233-5.

33. Janicek MF, Rosenshein NB. Invasive endometrial cancer in uteri resected for atypical endometrial hyperplasia. Gynecol Oncol 1994; 52: 373-8.

34. Miller C, Bidus MA, Pulcini JP, et al. The ability of endometrial biopsies with atypical complex hyperplasia to guide surgical management. Am J Obstet Gynecol 2008; 199: 69.e1-4.

35. Whyte JS, Gurney EP, Curtin JP, et al. Lymph node dissection in the surgical management of atypical endometrial hyperplasia. Am J Obstet Gynecol 2010; 202: 176.e1-4.

Received: 12.11 .2014 , accepted: 25.01 .2015 